\title{
Real Roots of Real Dirichlet L-Series ${ }^{1}$
}

\author{
By J. Barkley Rosser
}

\begin{abstract}
In the theory of the distribution of primes in arithmetic series, in assigning bounds in the three prime theorem, and in studying the class number of quadratic fields, a knowledge of the location of the real zeros of $L(s, \chi)$ is of value. A long standing conjecture is that there are no positive real zeros for any $k$. If proved, this result would be of value in each of the fields mentioned above. By a certain computational procedure the conjecture has already been verified for each individual $k \leq 67$. In the present paper, this earlier computational procedure was tried for each $k \leq 22 \overline{7}$ and failed for $k=163$. An improved computational procedure is given in the present paper, but still the case $k=163$ remained difficult. Finally, a new formula for $L(s, \chi)$ was discovered that made it possible to treat many values of $k$ simultaneously. By means of this formula, the difficult case of $k=163$ was finally treated adequately.
\end{abstract}

\section{Introduction}

Let $k$ be a positive integer. Let $\chi$ be a real, nonprincipal character $(\bmod k)$ and let

$$
L(s, \chi)=\sum_{n=1}^{\infty} \frac{\chi(n)}{n^{s}}
$$

By a computation using the methods of [1] it was shown that if $2 \leq k \leq 227$ and $k \neq 163$, then $L(s, \chi)$ has no positive real zeros. This computation was laid out by G. Gourrich. Computation on IBM equipment was furnished by Miss L. Cutler and E. Rea under the direction of E. C. Yowell. Computation on desk computers was furnished by Miss. L. Forthal and W. Paine under the direction of G. Blanch.

For $k=163$, the method of [1] definitely failed. It seems likely that by making a careful refinement of the estimates of [1] by means of an extensive computation, one could handle the case $k=163$. However, this did not seem a very profitable undertaking, and so a further study of $L(s, \chi)$ for positive real $s$ was made, and various alternative methods were devised. Some of these seem clearly superior to the method of [1]. One such superior method is a generalization of the method of Chowla (see [3]). Using this method and a table of characters $(\bmod k)$ prepared by Miss L. Cutler and E. Rea, it was a fairly quick matter for G. Gourrich to check that if $k \leq 227$ and $k \neq 163$, then $L(s, \chi)$ has no positive real zeros. Even by this method, the case $k=163$ remained very difficult. Probably the method will handle the case $k=163$, but it seemed clear that even by this method the case $k=163$ would require very extensive computations, and it seemed worthwhile to devise still further methods. This was done, and a method was finally found by which the case $k=163$ can be handled rather easily, with only a minor computation.

In the meantime, Chowla and Selberg (see [7]) have announced still another method for treating the case $k=163$.

One may conclude that it is now quite firmly established that if $k \leq 227$, then $L(s, \chi)$ has no positive real zeros.

\section{Generalization of Chowla's Method}

Throughout this section, we lay down the following conventions. $\chi$ is a real primitive character. $K$ shall be a positive integer, $\phi(n)$ shall be a function of positive integers which is periodic with period $K$, $L(s, \phi)$ shall denote the function that is got by analytic continuation from

$$
\sum_{n=1}^{\infty} \frac{\phi(n)}{n^{s}}
$$

so that for $R(s)>1$

$$
L(s, \phi)=\sum_{n=1}^{\infty} \frac{\phi(n)}{n^{s}},
$$

and $f(z, \phi)$ shall denote the function that is got by analytic continuation from

$$
\sum_{n=1}^{\infty} \phi(n) e^{-n 2}
$$

so that for $R(z)>0$,

$$
f(z, \phi)=\sum_{n=1}^{\infty} \phi(n) e^{-n z} .
$$

We cite without proof various results, the proofs for which can easily be derived from the results of chapter XIII of [5].

Theorem 1. For $s \neq 1$,

$$
L(s, \phi)=K^{-s} \sum_{n=1}^{K} \phi(n) \zeta\left(s, \frac{n}{K}\right) .
$$

Corollary. If

$$
\sum_{n=1}^{K} \phi(n)=0,
$$

then $L(s, \phi)$ is analytic for all $s$.

1 The preparation of this paper was sponsored (in part) by the Office of Nava! Research. 
Theorem 2. For all $z$ different from $2 \pi m i / K(m$ an integer)

$$
f(z, \phi)=\frac{1}{e^{K z}-1} \sum_{n=1}^{K} \phi(n) e^{(K-n) z} .
$$

Corollary. If

$$
\sum_{n=1}^{K} \phi(n)=0,
$$

then $f(z, \phi)$ is analytic for $|z|<2 \pi / K$, and

$$
f(0, \phi)=\sum_{n=1}^{K} \phi(n)\left(1-\frac{n}{K}\right) .
$$

Theorem 3. If $R(s)>1$, then

$$
\Gamma(s) L(s, \phi)=\int_{0}^{\infty} x^{s-1} f(x, \phi) d x .
$$

Corollary. If

$$
\sum_{n=1}^{K} \phi(n)=0,
$$

then for $R(s)>0$,

$$
\Gamma(s) L(s, \phi)=\int_{0}^{\infty} x^{s-1} f(x, \phi) d x .
$$

Since

$$
\sum_{n=1}^{k} \chi(n)=0
$$

the results stated in the three corollaries hold if we replace $\phi$ by $\chi$ and $K$ by $k$. In particular, if we can prove that $f(x, \chi) \geq 0$ for $x \geq 0$, it will follow from the corollary to theorem 3 that $L(s, \chi)$ has no positive real zeros. This can be readily proved for many values of $k$. The method of proof is as follows. With Chowla, we define

$$
\begin{aligned}
& \phi_{0}(n)=\phi(n) \\
& \phi_{r+1}(n)=\sum_{m=1}^{n} \phi_{r}(m) .
\end{aligned}
$$

We then have the following known result (see [4]).

Theorem 4. For $x>0$,

$$
f(x, \phi)=\left(1-e^{-x}\right)^{r} f\left(x, \phi_{r}\right) .
$$

Then, if there is an $r$ for which $\chi_{r}(n)$ is nonnegative for $n \geq 1$, we infer that $f(x, \chi) \geq 0$ for $x \geq 0$, and hence that $\bar{L}(s, \chi)$ has no positive real zeros.

For many $k$ 's, we can prove by a brief computation that there is an $r$ such that $\chi_{r}(n) \geq 0$ for $n \geq 1$. For a typical case, consider $k=53$ and let $\chi$ be the real nonprincipal character $(\bmod 53)$. In this case $\chi(n), \chi_{1}(n)$, and $\chi_{2}(n)$ are sometimes negative, but $\chi_{3}(n)$ is nonnegative for all positive $n$. The computations on which this statement is based are given in table 1 (at the end of the paper). The method of computation of table 1 is particularly simple, since from the definition of $\chi_{r+1}$ we have

$$
\chi_{r+1}(n+1)=\chi_{r+1}(n)+\chi_{r}(n+1) .
$$

So for $r \geq 1$ and $n \geq 2, \chi_{r}(n)$ is the sum of the numbers immediately above it and immediately to the left of it in table 1.

We have

$$
\chi_{1}(n+53)=\chi_{1}(53)+\sum_{m=54}^{n+53} \chi(m) .
$$

However $\chi_{1}(53)=0$ (see table 1 ) and $\chi$ is periodic with period 53 . So

$$
\chi_{1}(n+53)=\sum_{m=1}^{n} \chi(m)=\chi_{1}(n) .
$$

So $\chi_{1}(n)$ is periodic with period 53. Similarly, $\chi_{2}(n)$ is periodic with period 53 . However $\chi_{3}(53)=742$ (see table 1) so that

$$
\chi_{3}(n+53)=742+\chi_{3}(n) .
$$

As $\chi_{3}(n) \geq 0$ for $1 \leq n \leq 53$ (see table 1 ), it follows that $\chi_{3}(n) \geq 0$ for $n \geq 1$.

In table 2 , we have listed those $k$ 's $\leq 227$ for which there is a primitive $\chi$ and for which we could find an $r$ such that $\chi_{r}(n) \geq 0$ for $n \geq 1$. Opposite each $k$ is listed the least value of $r$ for which $\chi_{r}(n) \geq 0$ for $n \geq 1$. Opposite 8 in table 2 is given the $r$ corresponding to the character $\chi(1)=1, \quad \chi(3)=-1$, $\chi(5)=-1, \chi(7)=1$, and opposite $8^{*}$ is given the $r$ corresponding to the character $\chi(1)=1, \chi(3)=1$, $\chi(5)=-1, \chi(7)=-1$. The corresponding $\chi$ 's $(\bmod$ 24 ) are indicated by entries 24 and $24 *$ in table 2 . Similarly for 40 and $40^{*}, 56$ and $56^{*}$, etc.

It will be noted that so far we are using exactly Chowla's method (see [3]), though our justification for the method is different from Chowla's. As noted by Heilbronn in [4], there exist values of $k$ such that no $\chi_{r}(n)$ is nonnegative for every $n \geq 1$. In fact one can prove that $k=163$ is such a $k$; for actual computation for $k=163$ discloses that $f(\log (10 / 7), \chi)$ is negative, so that by theorem 4 thare cannot be any completely nonnegative $\chi_{r}$.

Our efforts to find an $r$ for the cases $k=43,67,88$, $123,148,173,187,188$, and 197 were sufficiently unrewarded that we suspect that for these values of $k$ also there is no $r$. At any rate we devised an improvement of Chowla's method to handle these intractable $k$ 's (except perhaps $k=163$ ).

Theorem 5. If

then

$$
F(s)=\int_{0}^{\infty} x^{s-1} f(x) d x
$$

$$
\left(1+\alpha r^{-s}\right) F(s)=\int_{0}^{\infty} x^{s-1}\{f(x)+\alpha f(r x)\} d x .
$$


Theorem 6. If $r$ is a positive integer and $\theta(n)$ is the coefficient of $n^{-s}$ in the Dirichlet series expansion of

then

$$
\left(1+\alpha r^{-s}\right) L(s, \phi),
$$

$$
f(x, \theta)=f(x, \phi)+\alpha f(r x, \phi) .
$$

We now illustrate the use of these theorems for the case $k=67$. Note that for $k=67, \chi(2)=\chi(3)=$ $\chi(5)=-1$. So

$$
\begin{aligned}
L(s, \chi) & =\Pi_{p}\left(1-\chi(p) p^{-s}\right)^{-1} \\
& =\frac{1}{\left(1+2^{-s}\right)\left(1+3^{-s}\right)\left(1+5^{-s}\right)_{p \geq 7}} \Pi_{p}\left(1-\chi(p) p^{-s}\right)^{-1} .
\end{aligned}
$$

So the Dirichlet series expansion of

$$
\left(1+2^{-s}\right) L(s, \chi)
$$

consists of the Dirichlet series expansion of $L(s, \chi)$ with all terms $n^{-s}$ removed for which $n$ is divisible by 2. Similarly, to get the Dirichlet series expansion of

$$
\left(1+2^{-s}\right)\left(1+3^{-s}\right) L(s, \chi),
$$

we remove all terms $n^{-s}$ for which $n$ is divisible by either 2 or 3 . Similarly for

$$
\left(1+2^{-s}\right)\left(1+3^{-s}\right)\left(1+5^{-s}\right) L(s, \chi) .
$$

We now verify by actual computation that $\chi_{2}(n) \geq 0$ for $n \geq 25$. Define $\phi(n)$ to be the coefficient of $n^{-s}$ in the Dirichlet series expansion of

Then

$$
\left(1+2^{-s}\right) L(s, \chi) \text {. }
$$

$$
\left(1+2^{-s}\right) \Gamma(s) L(s, \chi)=\int_{0}^{\infty} x^{s-1} f(x, \phi) d x
$$

and

Now

$$
f(x, \phi)=f(x, \chi)+f(2 x, \chi) .
$$

$$
\begin{aligned}
\left(1-e^{-x}\right)^{2} f\left(x, \phi_{2}\right)=f(x, \phi) & \\
= & f(x, \chi)+f(2 x, \chi) \\
& =\left(1-e^{-x}\right)^{2} f\left(x, \chi_{2}\right)+\left(1-e^{-2 x}\right)^{2} f\left(2 x, \chi_{2}\right) \\
& =\left(1-e^{-x}\right)^{2}\left\{f\left(x, \chi_{2}\right)+\left(1+e^{-x}\right)^{2} f\left(2 x, \chi_{2}\right)\right\}
\end{aligned}
$$

So

$$
f\left(x, \phi_{2}\right)=f\left(x, \chi_{2}\right)+\left(1+e^{-x}\right)^{2} f\left(2 x, \chi_{2}\right) .
$$

As each side of this is a power series in $e^{-x}$, corresponding coefficients must be equal. So

$$
\begin{aligned}
\phi_{2}(2 n) & =\chi_{2}(2 n)+\chi_{2}(n)+\chi_{2}(n-1) \\
\phi_{2}(2 n+1) & =\chi_{2}(2 n+1)+2 \chi_{2}(n) .
\end{aligned}
$$

Recalling that $\chi_{2}(n) \geq 0$ for $n \geq 25$, we see that surely $\phi_{2}(n) \geq 0$ for $n \geq 51$. Actual computation of $\phi_{2}(n)$ for $1 \leq n \leq 50$ discloses that actually $\phi_{2}(n) \geq 0$ for $n \geq 31$.

If we now define $\theta(n)$ as the coefficient of $n^{-s}$ in the Dirichlet series expansion of

then

$$
\left(1+2^{-s}\right)\left(1+3^{-s}\right) L(s, \chi),
$$

$$
\left(1+2^{-s}\right)\left(1+3^{-s}\right) \Gamma(s) L(s, \chi)=\int_{0}^{\infty} x^{-s} f(x, \theta) d x
$$

and

$$
f(x, \theta)=f(x, \phi)+f(3 x, \phi) .
$$

This latter equation gives a relation between $\theta_{2}$ and $\phi_{2}$. Using this relation with the result $\phi_{2}(n) \geq 0$ for $n \geq 31$ leads to the inference that $\theta_{2}(n) \geq 0$ for $n \geq 95$. Actual computation of $\theta_{2}(n)$ for $1 \leq n \leq 94$ discloses that actually $\theta_{2}(n) \geq 0$ for $n \geq 41$.

We proceed one step further, defining $\eta(n)$ as the coefficient of $n^{-8}$ in the Dirichlet series expansion of

Then

$$
\left(1+2^{-s}\right)\left(1+3^{-s}\right)\left(1+5^{-s}\right) L(s, \chi) .
$$

$\left(1+2^{-s}\right)\left(1+3^{-s}\right)\left(1+5^{-s}\right) \Gamma(s) L(s, \chi)=\int_{0}^{\infty} x^{s-1} f(s, \eta) d x$.

Also we find that $\eta_{2}(n) \geq 0$ for $n \geq 29$. We now ascertain by actual computation that $\eta_{3}(n) \geq 0$ for $n \leq 28$, and so conclude that $\eta_{3}(n) \geq 0$ for $n \geq 1$. Then $f(x, \eta) \geq 0$ for $x \geq 0$. So

$$
\left(1+2^{-s}\right)\left(1+3^{-s}\right)\left(1+5^{-s}\right) \Gamma(s) L(s, \chi)>0
$$

for $s>0$ and so $L(s, \chi)>0$ for $s>0$.

For other $k$ 's we proceeded similarly, except that when $\chi(-1)=1$ it was necessary to work with $\chi_{3}$, $\phi_{3}, \theta_{3}$, etc., instead of with $\chi_{2}, \phi_{2}, \theta_{2}$, etc., because the latter are periodic when $\chi(-1)=1$.

In table 3 we have listed against $k$ the combination

$$
\Pi\left(1+p_{i}^{-s}\right) L(s, \chi),
$$

which was used with that $k$ and also the least value of $r$ which sufficed with this combination.

We note in passing that we can handle the case $k=43$ with the combination

$$
\left(1+2^{-s}\right) L(s, \chi),
$$

but we need to take $r=9$, so that it is less laborious to take the combination

$$
\left(1+2^{-s}\right)\left(1+3^{-s}\right) L(s, \chi)
$$

for which $r=2$ suffices.

We suspect that this method will work for $k=163$. However for $k=163$, the combination

$$
\Pi\left(1+p_{i}^{-s}\right) L(s, \dot{\chi})
$$


will require at least five factors, and likely more than five, and the computations involved appear to be very extensive. So we sought other methods.

\section{Another Method}

We let $\chi$ be a real primitive character.

Define

$$
\begin{gathered}
\xi(s)=\pi^{-s / 2} \Gamma\left(\frac{s}{2}\right) \zeta(s) \\
\xi(s, \chi)=\left(\frac{\pi}{k}\right)^{-s / 2} \Gamma\left(\frac{s}{2}\right) L(s, \chi) \\
\text { if } \chi(-1)=1, \\
\xi(s, \chi)=\left(\frac{\pi}{k}\right)^{-(s+1) / 2} \Gamma\left(\frac{s+1}{2}\right) L(s, \chi) \\
\text { if } \chi(-1)=-1 .
\end{gathered}
$$

We have the known results

$$
\begin{gathered}
\xi(s)=\xi(1-s), \\
\xi(s, \chi)=\xi(1-s, \chi) .
\end{gathered}
$$

Also known is:

Theorem 7. If $k_{1}$ and $k_{2}$ are any real constants, then for $k_{1} \leq \sigma \leq k_{2}$,

$$
\xi(s)-\frac{1}{s(s-1)}
$$

and $\xi(s, \chi)$ are bounded.

Theorem 8. There is a function $f(x)$ with the following properties:

(I) $f(x)$ is analytic for $R(x)>0$.

(II) $f(x)$ is positive for $x>0$.

(III) $f(x)$ is monotone decreasing for $x>0$.

(IV) For $x \geq 1$,

$$
f(x) \leq \exp \left(-\frac{2 \pi x}{\sqrt{k}}\right) f(1) \exp \left(\frac{2 \pi}{\sqrt{k}}\right) .
$$

(V) There is a positive constant $A$ such that for $x>0$,

$$
\frac{A}{\sqrt{4 x+1}} \exp \left(-\frac{2 \pi x}{\sqrt{k}}\right) \leq f(x) .
$$

(VI) For $\sigma>\sigma_{k}$,

$$
\xi(s) \xi(s, \chi)=\int_{0}^{\infty} x^{s-1} f(x) d x .
$$

Proof. Define $c_{n}$ as the coefficient of $n^{-s}$ in the Dirichlet series expansion of $\zeta(s) L(s, \chi)$, so that for $\sigma>1$,

$$
\zeta(s) L(s, \chi)=\sum_{n=1}^{\infty} \frac{c_{n}}{n^{s}}
$$

Then $c_{1}=1$ and $c_{n} \geq 0$.

Case 1. $\quad \chi(-1)=1$. We have

$$
\left(\frac{\pi}{\sqrt{k}}\right)^{-s / 2} \Gamma\left(\frac{s}{2}\right) n^{-s / 2}=\int_{0}^{\infty} v^{s / 2} \exp \left(-\frac{\pi n v}{\sqrt{k}}\right) \frac{d v}{v} .
$$

So

$$
\begin{aligned}
& \pi^{-s / 2} \Gamma\left(\frac{s}{2}\right)\left(\frac{\pi}{k}\right)^{-s / 2} \Gamma\left(\frac{s}{2}\right) n^{-s} \\
& =\int_{0}^{\infty} \int_{0}^{\infty}(v w)^{s / 2} \exp \left(-\frac{\pi n(v+w)}{\sqrt{k}}\right) \frac{d v}{v} \frac{d w}{w} .
\end{aligned}
$$

Multiplying by $c_{n}$ and summing gives for $\sigma>\sigma_{k}$

$$
\xi(s) \xi(s, \chi)
$$

$$
=\int_{0}^{\infty} \int_{0}^{\infty}(v w)^{s / 2}\left\{\sum_{n=1}^{\infty} c_{n} \exp \left(-\frac{\pi n(v+w)}{\sqrt{k}}\right)\right\} \frac{d v}{v} \frac{d w}{w} .
$$

We now treat the integral on the right as a double integral over the first quadrant. Since the integrand is symmetric in $v$ and $w$, we may replace the integral by twice the integral over the area in the first quadrant below the $45^{\circ}$ ray thru the origin. In this, we introduce

$$
\begin{aligned}
& x=\sqrt{v w} \\
& y=v+w
\end{aligned}
$$

as new variables of integration, getting

$$
\begin{aligned}
& \xi(s) \xi(s, \chi) \\
= & 4 \int_{0}^{\infty} x^{s-1} d x \int_{2 x}^{\infty}\left\{\sum_{n=1}^{\infty} c_{n} \exp \left(-\frac{\pi n v}{\sqrt{k}}\right)\right\} \frac{d y}{\sqrt{y^{2}-4 x^{2}}} .
\end{aligned}
$$

We take

$$
f(x)=4 \int_{2 x}^{\infty}\left\{\sum_{n=1}^{\infty} c_{n} \exp \left(-\frac{\pi n y}{\sqrt{k}}\right)\right\} \frac{d y}{\sqrt{y^{2}-4 x^{2}}}
$$

and conclude (II) and (VI) of our theorem. We put $y=2 x+z$ in eq 6 and get

$$
f(x)=4 \int_{0}^{\infty}\left\{\sum_{n=1}^{\infty} c_{n} \exp \left(-\frac{\pi n(2 x+z)}{\sqrt{k}}\right)\right\} \frac{d z}{\sqrt{z} \sqrt{4 x+z}} .
$$

Then (I) and (III) of our theorem are evident. Further, for $x \geq 1$, we write 


$$
\begin{aligned}
f(x) & =4 \exp \left(-\frac{2 \pi x}{\sqrt{k}}\right) \int_{0}^{\infty}\left\{\sum_{n=1}^{\infty} c_{n} \exp \left(-\frac{\pi n(2 x+z)}{\sqrt{k}}+\frac{2 \pi x}{\sqrt{k}}\right)\right\} \frac{d z}{\sqrt{z} \sqrt{4 x+z}} \\
& \leq 4 \exp \left(-\frac{2 \pi x}{\sqrt{k}}\right) \int_{0}^{\infty}\left\{\sum_{n=1}^{\infty} c_{n} \exp \left(-\frac{\pi n(2+z)}{\sqrt{k}}+\frac{2 \pi}{\sqrt{k}}\right)\right\} \frac{d z}{\sqrt{z} \sqrt{4+z}} \\
& \leq \exp \left(-\frac{2 \pi x}{\sqrt{k}}\right)\left\{f(1) \exp \left(\frac{2 \pi}{\sqrt{k}}\right)\right\}
\end{aligned}
$$

So (IV) holds.

By eq 7, using only the first term of the series, we get

$$
\begin{aligned}
f(x) & \geq 4 \int_{0}^{\infty} \exp \left(-\frac{\pi(2 x+z)}{\sqrt{k}}\right) \frac{d z}{\sqrt{z} \sqrt{4 x+z}} \\
& \geq 4 \int_{0}^{1} \exp \left(-\frac{\pi(2 x+z)}{\sqrt{k}}\right) \frac{d z}{\sqrt{z} \sqrt{4 x+z}} \\
& \geq \frac{4}{\sqrt{4 x+1}} \exp \left(-\frac{2 \pi x}{\sqrt{k}}\right) \int_{0}^{1} \exp \left(-\frac{\pi z}{\sqrt{k}}\right) \frac{d z}{\sqrt{z}}
\end{aligned}
$$

So (V) holds.

In the relation

$$
K_{0}(z)=\int_{0}^{\infty} \exp (-z \cosh \phi) d \phi
$$

(see [5], p. 384, Example 40) put $\cosh \phi=1+t$. There results

$$
K_{0}(z)=\int_{0}^{\infty} \exp (-z(1+t)) \frac{d t}{\sqrt{t^{2}+2 t}} .
$$

So for $x>0$

$$
4 K_{0}\left(\frac{2 \pi n x}{\sqrt{k}}\right)=4 \int_{0}^{\infty} \exp \left(-\frac{2 \pi n x(1+t)}{\sqrt{k}}\right) \frac{d t}{\sqrt{t^{2}+2 t}} .
$$

Putting $t=z / 2 x$, we get

$$
4 K_{0}\left(\frac{2 \pi n x}{\sqrt{k}}\right)=4 \int_{0}^{\infty} \exp \left(-\frac{\pi n(2 x+z)}{\sqrt{k}}\right) \frac{d z}{\sqrt{k} \sqrt{4 x+z}} .
$$

So by eq 7 ,

$$
f(x)=4 \sum_{n=1}^{\infty} c_{n} K_{0}\left(\frac{2 \pi n x}{\sqrt{k}}\right) .
$$

Case 2. $\chi(-1)=-1$. By the duplication formula for the gamma function

$$
\begin{aligned}
& \pi^{-s / 2} \Gamma\left(\frac{s}{2}\right)\left(\frac{\pi}{k}\right)^{-(s+1) / 2} \Gamma\left(\frac{s+1}{2}\right) n^{-s} \\
& =2 \sqrt{k}\left(\frac{2 \pi}{\sqrt{k}}\right)^{-s} \Gamma(s) n^{-s} \\
& =2 \sqrt{k} \int_{0}^{\infty} x^{s-1} \exp \left(-\frac{2 \pi n x}{\sqrt{k}}\right) d x
\end{aligned}
$$

Multiplying by $c_{n}$ and summing gives for $\sigma>\sigma_{k}$

$$
\xi(s) \xi(s, \chi)=2 \sqrt{k} \int_{0}^{\infty} x^{s-1}\left\{\sum_{n=1}^{\infty} c_{n} \exp \left(-\frac{2 \pi n x}{\sqrt{k}}\right)\right\} d x .
$$

We now take

$$
f(x)=2 \sqrt{k} \sum_{n=1}^{\infty} c_{n} \exp \left(-\frac{2 \pi n x}{\sqrt{k}}\right)
$$

and conclude (I), (II), (III), and (VI) of our theorem. We can easily show that for $x \geq 1$,

$$
f(x) \leq \exp \left(-\frac{2 \pi x}{\sqrt{k}}\right)\left\{f(1) \exp \left(\frac{2 \pi}{\sqrt{k}}\right)\right\}
$$

and so (IV) holds. Using the first term of eq 8 gives

$$
f(x)>2 \sqrt{k} \exp \left(-\frac{2 \pi x}{\sqrt{k}}\right) .
$$

So we may take $A=2 \sqrt{k}$ in $(\mathrm{V})$.

Theorem 9. For all $s$

$$
\xi(s) \xi(s, \chi)=\frac{\xi(1, \chi)}{s(s-1)}+\int_{1}^{\infty}\left(x^{s-1}+x^{-s}\right) f(x) d x .
$$

Proof. Use the method of Heilbronn (see [8]).

Theorem 10. For $R(x)>0$,

$$
f\left(\frac{1}{x}\right)=x f(x)+(x-1) \xi(1, \chi) .
$$

Proof. Comparing (VI) of theorem 8 with theorem gives for $\sigma>\sigma_{k}$

$$
\int_{0}^{1} x^{s-1} f(x) d x=\frac{\xi(1, \chi)}{s(s-1)}+\int_{1}^{\infty} x^{-s} f(x) d x .
$$

We can rewrite this as

$$
\begin{gathered}
\int_{0}^{1} x^{s-1} f(x) d x=\xi(1, \chi) \int_{0}^{1} x^{s-2} d x \\
-\xi(1, \chi) \int_{0}^{1} x^{s-1} d x+\int_{0}^{1} x^{s-2} f\left(\frac{1}{x}\right) d x \\
=\int_{0}^{1} x^{s-1}\left\{\xi(1, \chi)\left(\frac{1}{x}-1\right)+\frac{1}{x} f\left(\frac{1}{x}\right)\right\} d x .
\end{gathered}
$$


Replacing $x$ by $e^{-t}$ in these integrals and using the uniqueness theorem for Laplace transforms, we infer that for $0<x<1$

$$
f(x)=\xi(1, \chi)\left(\frac{1}{x}-1\right)+\frac{1}{x} f\left(\frac{1}{x}\right) .
$$

So our theorem holds for $0<x<1$. Then by analytic continuation, it holds for $R(x)>0$.

If we let $x \rightarrow \infty$ in this result, we have $x f(x) \rightarrow 0$ and

$$
f\left(\frac{1}{x}\right)>f(1)>0
$$

So we have an alternative proof of the known result that $\xi(1, \chi)>0$. If we differentiate both sides and put $x=1$, we infer:

Theorem 11.

$$
\xi(1, \chi)=-f(1)-2 f^{\prime}(1) .
$$

Another consequence of theorem 10 is that

$$
f(x)=0\left(\frac{1}{x}\right)
$$

as $x \rightarrow 0$. Hence in (VI) of theorem 8 , we can take $\sigma_{k}=1$.

Theorem 12. For $0<s<1$,

$$
-\xi(s) \xi(s, \chi)=\int_{1}^{\infty}\left(x^{s-2}+x^{-s-1}\right)\{\xi(1, \chi)-x f(x)\} d x .
$$

Proof. Use theorem 9.

It is clear from this that if $x f(x) \leq \xi(1, \chi)$ for $1 \leq x$, then $L(s, \chi)>0$ for $s>0$. There are many $k$ 's for which $x f(x) \leq \xi(1, \chi)$ for $1 \leq x$. For example, let $\chi(-1)=-1$ and $k \leq 39$. Then

$$
\frac{2 \pi}{\sqrt{k}}>1
$$

Hence by eq 8

$$
\begin{aligned}
& f(x)=2 \sqrt{k} \sum_{n=1}^{\infty} c_{n} \exp \left(-\frac{2 \pi n x}{\sqrt{k}}\right), \\
& x f(x)=2 \sqrt{k} \sum_{n=1}^{\infty} c_{n} x \exp \left(-\frac{2 \pi n x}{\sqrt{k}}\right) .
\end{aligned}
$$

However, with $2 \pi / \sqrt{k}>1$,

$$
x \exp \left(-\frac{2 \pi n x}{\sqrt{k}}\right)
$$

is decreasing for $x>1$. So $x f(x)$ is a decreasing function for $x \geq 1$. So we have only to prove

$$
f(1)<\xi(1, \chi) \text {. }
$$

So, by theorem 11 , it suffices to prove

$$
-f^{\prime}(1)>f(1) \text {. }
$$

However

$$
\begin{aligned}
-f^{\prime}(1) & =2 \sqrt{k} \sum_{n=1}^{\infty} c_{n} \frac{2 \pi n}{\sqrt{k}} \exp \left(-\frac{2 \pi n}{\sqrt{k}}\right) \\
& >2 \sqrt{k} \sum_{n=1}^{\infty} c_{n} \exp \left(-\frac{2 \pi n}{\sqrt{k}}\right) \\
& >f(1) .
\end{aligned}
$$

If $\chi(-1)=1$, one can carry out similar reasoning based on eq 7 , since

$$
\int_{0}^{\infty} \exp \left(-\frac{\pi n z}{\sqrt{k}}\right) \frac{d z}{\sqrt{z} \sqrt{4 x+z}}
$$

is a decreasing function of $x$.

Thus we conclude by a very simple reasoning, which does not even involve inspection of the values of $\chi$, that for $k \leq 39, L(s, \chi)$ has no positive real zeros.

For larger $k$ 's, we would need to know the values of $\chi(n)$ for some of the smaller values of $n$. However, usually a knowledge of the values of $\chi(n)$ for $n \leq \sqrt{k}$ would be more than ample.

Unfortunately, this method is not general. In particular, it fails for $k=163$. Indeed (as we will show in the next section) for $k=163$,

$$
f(1)>\xi(1, \chi)
$$

so that it is impossible to have $x f(x) \leq \xi(1, \chi)$ for $x \geq 1$. So for $k=163$, more subtle methods are required.

\section{Treatment of $k=163$}

Throughout this section, let $k=163$.

Then $\chi(-1)=-1$. Also, the class number of $\sqrt{-k}$ is unity, so that

$$
\xi(1, \chi)=\sqrt{k} .
$$

Temporarily define

$$
g(x)=\xi(1, \chi)-x f(x) .
$$

Lemma 1. For $1 \leq x \leq \sqrt{k} / 2 \pi, g(x)<0$.

Proof. Using the first term of eq 8 , we get

As

$$
\begin{array}{r}
g(x)<\xi(1, \chi)-2 \sqrt{k} x \exp \left(-\frac{2 \pi x}{\sqrt{k}}\right) \\
<\sqrt{k}\left(1-2 x \exp \left(-\frac{2 \pi x}{\sqrt{k}}\right)\right) .
\end{array}
$$

$$
x \exp \left(-\frac{2 \pi x}{\sqrt{k}}\right)
$$


is increasing for $1 \leq x<\sqrt{k} / 2 \pi$, it suffices to prove

$$
\frac{1}{2}<\exp \left(-\frac{2 \pi}{\sqrt{k}}\right) \text {. }
$$

However, for $k=163$,

$$
\exp \left(-\frac{2 \pi}{\sqrt{k}}\right)=0.61,
$$

and our lemma is proved.

Lemma 2. For $(\sqrt{k} / 2 \pi) \leq x, g^{\prime}(x)>0$.

Proof. For $(\sqrt{k} / 2 \pi) \leq x$,

$$
\frac{d}{d x}\left\{x \exp \left(-\frac{2 \pi x}{\sqrt{k}}\right)\right\} \leq 0
$$

and for $n>1$

$$
\frac{d}{d x}\left\{x \exp \left(-\frac{2 \pi n x}{\sqrt{k}}\right)<0 .\right.
$$

Multiplying by $2 c_{n} \sqrt{k}$ and summing gives

$$
\frac{d}{d x}\{x f(x)\}<0
$$

for $(\sqrt{k} / 2 \pi) \leq x$. This establishes our lemma.

Lemma 3 . There is a number $a$ such that:

(I) $1<a$.

(II) For $1 \leq x<a, g(x)<0$.

(III) For $a<x, g(x)>0$.

Proof. Obvious by lemma 1, lemma 2, and the fact that

$$
\operatorname{Lim}_{x \rightarrow \infty} g(x)=\xi(1, \chi)>0 .
$$

Indeed, we have $a>\sqrt{k} / 2 \pi$, but this fact is not needed.

Lemma 4. For $1 / 2 \leq s<1$,

$$
-\frac{\xi(s) \xi(s, \chi)}{a^{s-1}+a^{-s}}
$$

is a monotone, nondecreasing function of $s$.

Proof. By theorem 12,

$$
-\frac{\xi(s) \xi(s, \chi)}{a^{s-1}+a^{-s}}=\int_{1}^{\infty} \frac{x^{s-1}+x^{-s}}{a^{s-1}+a^{-s}} \frac{g(x)}{x} d x .
$$

So

$$
\frac{\partial}{\partial s}\left\{-\frac{\xi(s) \xi(s, \chi)}{a^{s-1}+a^{-s}}\right\}=\int_{1}^{\infty} \frac{\partial}{\partial s}\left\{\frac{x^{s-1}+x^{-s}}{a^{s-1}+a^{-s}}\right\} \frac{g(x)}{x} d x .
$$

However,

$$
\begin{aligned}
\frac{\partial}{\partial s}\left\{\frac{x^{s-1}+x^{-s}}{a^{s-1}+a^{-s}}\right\} & =\frac{\left((a x)^{2 s-1}-1\right)(\log x-\log a)}{(a x)^{s}\left(a^{s-1}+a^{-s}\right)^{2}} \\
& +\frac{\left(x^{2 s-1}-a^{2 s-1}\right)(\log x+\log a)}{(a x)^{s}\left(a^{s-1}+a^{-s}\right)^{2}} .
\end{aligned}
$$

So for $1 / 2<s$, we see that

$$
\frac{\partial}{\partial s}\left\{\frac{x^{s-1}+x^{-s}}{a^{s-1}+a^{-s}}\right\}
$$

is negative if $1 \leq x<a$ and positive if $a<x$. So by lemma 3 , we see that for $1 / 2 \leq \mathrm{s}$

$$
\frac{\partial}{\partial s}\left\{\frac{x^{s-1}+x^{-s}}{a^{s-1}+a^{-s}}\right\} \frac{g(x)}{x}
$$

is nonnegative for $1 \leq x$. Thus our lemma is proved. If we can prove that the function

$$
-\frac{\xi(s) \xi(s, \chi)}{a^{s-1}+a^{s}}
$$

is positive at $s=1 / 2$, we can conclude that it is positive for $s \geq 1 / 2$. As it is unchanged by replacing $s$ by $1-s$, we could conclude that it is positive for $0<s<1$. So we have proved:

Lemma 5. If $L(s, \chi)$ is positive for $s=1 / 2$, then it is positive for $s>0$.

We have now reduced the problem to that of proving that $L(1 / 2, \chi)>0$. This result is proved in [7]. Alternatively, one can compute $L(1 / 2, \chi)$ explicitly by means of the formula

$$
L\left(\frac{1}{2}, \chi\right)=\frac{2\left(\frac{\pi}{163}\right)^{\frac{3}{4}}}{\Gamma\left(\frac{3}{4}\right)} \sum_{n=1}^{\infty} n \chi(n) \int_{1}^{\infty} x^{-1 / 4} \exp \left(-\frac{n^{2} \pi x}{163}\right) d x
$$

This is not exactly an agreeable computation, but by using a table of the incomplete gamma function, it is not unduly laborious. The computation was carried out by Miss S. Marks under the direction of $\mathrm{G}$. Blanch, and the value

$$
L\left(\frac{1}{2}, \chi\right)=0.06910
$$

was obtained.

A shorter computation results if we use theorem 9. Putting $s=1 / 2$ in this, we see that it suffices to prove that

$$
-4 \xi(1, \chi)+2 \int_{1}^{\infty} x^{-1 / 2} f(x) d x
$$

is negative. That is, using eq 8 and eq 9 , it suffices to prove

$$
1>\sum_{n=1}^{\infty} c_{n} \int_{0}^{\infty} x^{-1 / 2} \exp \left(-\frac{2 \pi n x}{\sqrt{k}}\right) d x .
$$

That is, it suffices to prove

$$
\frac{1}{\sqrt{2 \sqrt{k}}}>\sum_{n=1}^{\infty} \frac{c_{n}}{\sqrt{n}} \operatorname{erfc}\left(2 \sqrt{\frac{\pi n}{\sqrt{k}}}\right)
$$


where

$$
\operatorname{erfc}(y)=\frac{1}{\sqrt{2 \pi}} \int_{y}^{\infty} \exp \left(-\frac{1}{2} t^{2}\right) d t
$$

Now for $k=163$, we have $\chi(p)=-1$ for every prime $p$ with $2 \leq p \leq 37$. So for $n \leq 40, c_{n}=1$ when $n$ is a perfect square, and zern otherwise. So we need only show

$$
\begin{gathered}
\frac{1}{\sqrt{2 \sqrt{k}}}>\operatorname{erfc}\left(2 \sqrt{\frac{\pi}{\sqrt{k}}}\right)+\frac{1}{2} \operatorname{erfc}\left(4 \sqrt{\frac{\pi}{\sqrt{k}}}\right) \\
+\frac{1}{3} \operatorname{erfc}\left(6 \sqrt{\frac{\pi}{\sqrt{k}}}\right)+\cdots
\end{gathered}
$$

that is, we need only show

$$
\begin{aligned}
0.1979> & \operatorname{erfc}(0.9921)+\frac{1}{2} \operatorname{erfc}(2(0.9921)) \\
& +\frac{1}{3} \operatorname{erfc}(3(0.9921))+\cdots
\end{aligned}
$$

Using a four-place table of erfc, we get

$$
\begin{aligned}
\operatorname{erfc}(0.9921) & =0.1606 \\
\frac{1}{2} \operatorname{erfc}(2(0.9921)) & =0.0118 \\
\frac{1}{3} \operatorname{erfc}(3(0.9921)) & =0.0005 \\
\frac{1}{4} \operatorname{erfc}(4(0.0021)) & =0.0000
\end{aligned}
$$

As $0.1979>0.1729$, we conclude that $L(1 / 2, \chi)>0$.

\section{Miscellaneous Results}

If $L(s, \chi)$ is to be positive for $0<s$, then from

$$
\Gamma(s) L(s, \chi)=\int_{0}^{\infty} x^{s-1} f(x, \chi) d x
$$

we see that

$$
\int_{0}^{\infty} x^{s-1} f(x, \chi) d x
$$

must be positive even when $f(x, \chi)$ is negative for some $x$ (as when $k=163$ ). In an effort to prove this for various $k$ 's, we undertook to find more about the behavior of $f(x, \chi)$. Nothing particularly useful for our purpose was discovered. However miscellaneous results were found, and we list them without proof.

Theorem 13. For $s \neq 1$,

$$
L(s, \phi)=-\frac{\Gamma(1-s)}{2 \pi i} \int(-z)^{s-1} f(z, \phi) d z
$$

where the contour begins at $+\infty$ on the real axis, encircles the origin counterclockwise once, and returns to $+\infty$ on the real axis, and where the contour does not encircle any of the points $\pm 2 \pi \mathrm{mi} / \mathrm{K}$ ( $m$ a positive integer), and where $|\arg (-z)| \leq \pi$.

Theorem 14. If

$$
\sum_{n=1}^{k} \phi(n)=0,
$$

then

Define

$$
f^{(m)}(0, \phi)=(-1)^{m} L(-m, \phi) .
$$

$$
T_{m}=T(m, K, \phi)=\sum_{r=1}^{K} \phi(r)\left(1-\frac{r}{K}\right)^{m} .
$$

Theorem 15. For $\boldsymbol{M} \geq 1$,

$$
T_{M}=\frac{(-1)^{M}}{K^{M}} \sum_{N=0}^{M}(-1)^{N} \phi_{N+1}(K) \Delta^{N}\left(1^{M}\right),
$$

where $\Delta^{N}\left(1^{M}\right)$ denotes the $N$-th difference of $x^{M}$ evaluated at $x=1$. That is

$$
\Delta^{N}\left(1^{M}\right)=(-1)^{N} \sum_{r=0}^{N}(-1)^{r} \frac{N !}{(N-r) ! r !}(1+r)^{M} .
$$

Theorem 16. If

$$
\sum_{n=1}^{K} \phi(n)=0
$$

then

$$
\begin{aligned}
f^{(m)}(0, \phi)= & \frac{(m !) K^{m}}{(m+1) !}\left\{T_{m+1}-\frac{m+1}{2} T_{m}+\frac{(m+1)_{m}}{2 !} B_{1} T_{m-1}\right. \\
& \left.-\frac{(m+1)(m)(m-1)(m-2)}{4 !} B_{2} T_{m-3}+\cdots\right\}
\end{aligned}
$$

where $B_{m}$ is the $m$-th Bernoulli number as defined on p. 125 of [5].

Theorem 17 . For $x$ positive and small

$$
f(x, \phi)=e^{-x} \sum_{r=0}^{\infty} A_{r}\left(1-e^{-x}\right)^{r},
$$

where

$$
A_{r}=\frac{1}{r !}\left[\left(\frac{d}{d x}+1\right)\left(\frac{d}{d x}+2\right) \cdots\left(\frac{d}{d x}+r\right) f(x, \phi)\right]_{x=0} .
$$

Theorem 18. If $\chi$ is a primitive real character and $\chi(-1)=1$, then:

(1) For $|z|<\frac{2 \pi}{k}$,

$$
f(z, \chi)=\frac{\sqrt{k}}{\pi} \sum_{m=0}^{\infty}(-1)^{m}\left(\frac{k z}{2 \pi}\right)^{2 m+1} L(2+2 m, \chi) .
$$


(2) For $|z|<\frac{2 \pi R}{k}, z \neq \frac{2 \pi m i}{k}$,

$$
f(z, \chi)=\frac{\sqrt{k}}{\pi} \sum_{r=1}^{\infty} \frac{\chi(r)}{r} \frac{1}{1+\left(\frac{k z}{2 \pi r}\right)^{2}} .
$$

$f(z, \chi)=\frac{\sqrt{k}}{\pi} \frac{k z}{2 \pi} \sum_{r=1}^{R-1} \frac{\chi(r)}{r^{2}} \frac{1}{1+\left(\frac{k z}{2 \pi r}\right)^{2}}$

(4) For $z \neq \frac{2 \pi m i}{k}$,

$$
+\frac{\sqrt{k}}{\pi} \sum_{m=0}^{\infty}(-1)^{m}\left(\frac{k z}{2 \pi}\right)^{2 m+1}\left\{L(2+2 m, \chi)--\sum_{r=1}^{R-1} \frac{\chi(r)}{r^{2+2 m}}\right\}
$$

$$
\begin{array}{r}
f(z, \chi)=\frac{\sqrt{k}}{\pi} \sum_{m=0}^{M}(-1)^{m}\left(\frac{k z}{2 \pi}\right)^{2 m+1} L(1+2 m, \chi) \\
+(-1)^{M} \frac{\sqrt{k}}{2 \pi}\left(\frac{k z}{2 \pi}\right)^{2 M} \sum_{r=1}^{\infty} \frac{\chi(r)}{r^{1+2 M}} \frac{1}{1+\left(\frac{k z}{2 \pi r}\right)^{2}} .
\end{array}
$$

(3) For $z \neq \frac{2 \pi m i}{k}$,

$$
f(z, \chi)=\frac{\sqrt{k}}{\pi} \frac{k z}{2 \pi} \sum_{r=1}^{\infty} \frac{\chi(r)}{r^{2}} \frac{1}{1+\left(\frac{k z}{2 \pi r}\right)^{2}} .
$$

TABLE 1. Characters and sums of characters $(\bmod 53)$

(4) For $z \neq \frac{2 \pi m i}{k}$,

$$
\begin{aligned}
f(z, \chi)= & \frac{\sqrt{k}}{\pi} \sum_{m=0}^{M-1}(-1)^{m}\left(\frac{k z}{2 \pi}\right)^{2 m+1} L(2+2 m, \chi) \\
& +(-1)^{M} \frac{\sqrt{k}}{\pi}\left(\frac{k z}{2 \pi}\right)^{2 M+1} \sum_{r=1}^{\infty} \frac{\chi(r)}{r^{2+2 M}} \frac{1}{1+\left(\frac{k z}{2 \pi r}\right)^{2}} .
\end{aligned}
$$

Theorem 19. If $\chi$ is a primitive real character and $\chi(-1)=-1$, then:

(1) For $|z|<\frac{2 \pi}{k}$,

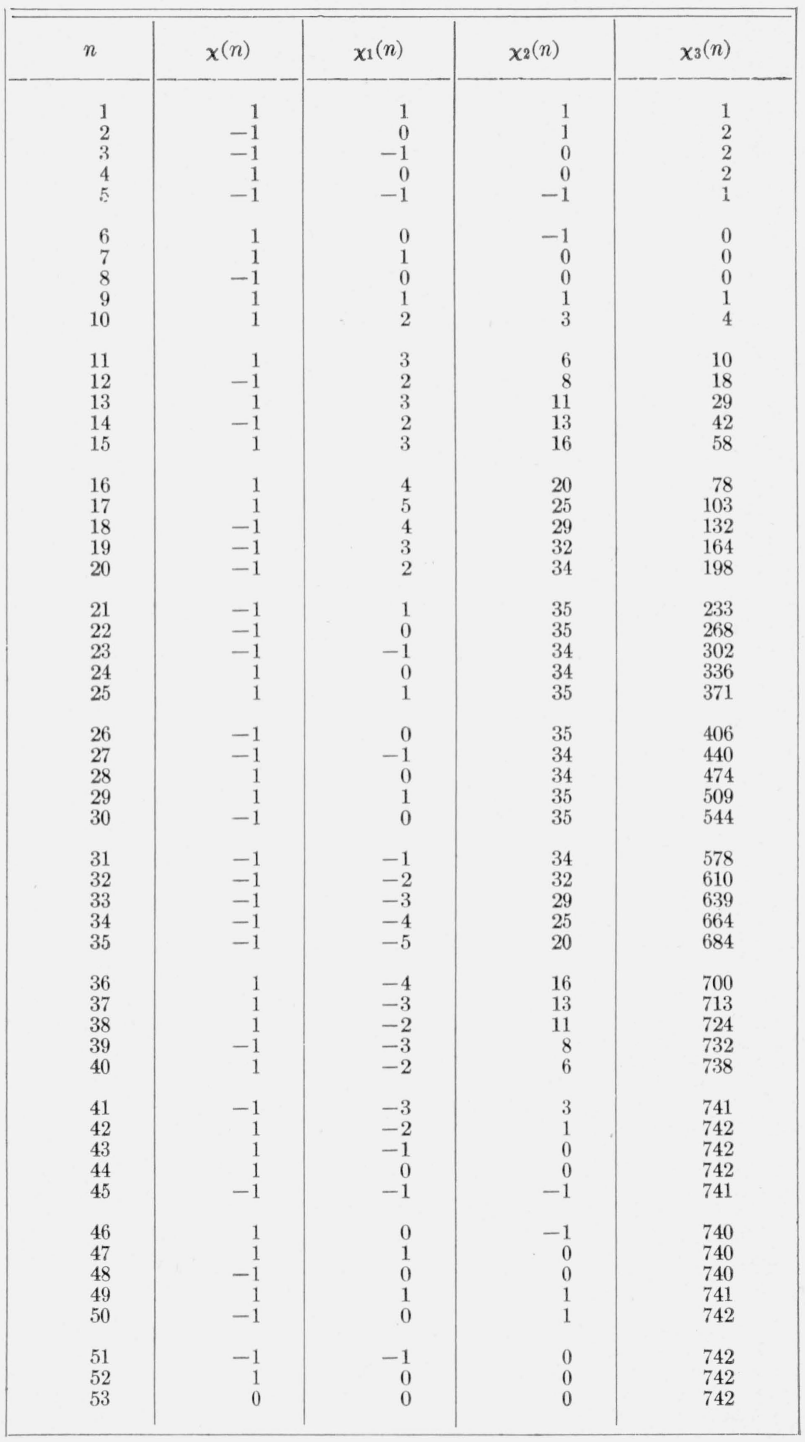

$$
\begin{aligned}
& f(z, \chi)=\frac{\sqrt{k}}{\pi} \sum_{r=1}^{R-1} \frac{\chi(r)}{r} \frac{1}{1+\left(\frac{k z}{2 \pi r}\right)^{2}} \\
& +\frac{\sqrt{k}}{\pi} \sum_{m=0}^{\infty}(-1)^{m}\left(\frac{k z}{2 \pi}\right)^{2 m}\left\{L(1+2 m, \chi)-\sum_{r=1}^{R-1} \frac{\chi(r)}{r^{1+2 m}}\right\} .
\end{aligned}
$$

(3) For $z \neq \frac{2 \pi m i}{k}$,

513 
TABLE 2. Values of $k$ and corresponding values of $r$

\begin{tabular}{|c|c|c|c|c|c|c|c|}
\hline$k$ & $r$ & $k$ & $r$ & $k$ & $r$ & $k$ & $r$ \\
\hline 3 & 1 & 55 & 1 & 109 & 2 & 165 & 2 \\
\hline 4 & 1 & 56 & 1 & 111 & 1 & 167 & 1 \\
\hline $\begin{array}{l}5 \\
7\end{array}$ & $\begin{array}{l}2 \\
1\end{array}$ & $\begin{array}{l}56^{*} \\
57\end{array}$ & $\begin{array}{l}2 \\
2\end{array}$ & $\begin{array}{l}113 \\
115\end{array}$ & $\begin{array}{l}2 \\
4\end{array}$ & $\begin{array}{l}168 \\
168^{*}\end{array}$ & 2 \\
\hline & $\begin{array}{l}1 \\
2\end{array}$ & & 1 & $\begin{array}{l}100 \\
116\end{array}$ & $\begin{array}{l}4 \\
1\end{array}$ & 172 & 2 \\
\hline $8^{*}$ & 1 & 60 & 2 & 119 & 1 & 177 & 2 \\
\hline 11 & 1 & 61 & 2 & 120 & 1 & 179 & 2 \\
\hline 12 & 2 & 65 & 2 & $120^{*}$ & 2 & 181 & 2 \\
\hline 13 & 2 & 68 & 1 & 124 & 2 & 183 & 1 \\
\hline 15 & & 69 & 2 & 127 & 2 & 184 & 2 \\
\hline 17 & 2 & 71 & 1 & 129 & 2 & $184^{*}$ & 2 \\
\hline $\begin{array}{l}19 \\
? 0\end{array}$ & 2 & $\begin{array}{l}73 \\
76\end{array}$ & 2 & 131 & 1 & $\begin{array}{l}185 \\
189\end{array}$ & \\
\hline & $\begin{array}{l}1 \\
2\end{array}$ & 76 & $\frac{2}{7}$ & 132 & 1 & 191 & $\frac{1}{2}$ \\
\hline $\begin{array}{l}21 \\
23\end{array}$ & 1 & 79 & 1 & 133 & 2 & 193 & 2 \\
\hline 23 & & 79 & & 136 & 2 & 195 & 2 \\
\hline 24 & 1 & 83 & 1 & $136^{*}$ & 1 & 199 & 1 \\
\hline $24^{*}$ & 2 & 84 & 1 & 137 & 2 & 201 & 2 \\
\hline 28 & 2 & $\begin{array}{l}85 \\
87\end{array}$ & 2 & 139 & 2 & 203 & 2 \\
\hline 29 & 2 & 87 & 1 & 140 & 2 & 204 & 2 \\
\hline 31 & 1. & $88^{*}$ & 2 & 141 & 2 & 205 & 2 \\
\hline 33 & 2 & 89 & 2 & 143 & 1 & 209 & 2 \\
\hline 35 & 1 & 91 & 3 & 145 & 2 & 211 & 3 \\
\hline 37 & 2 & 92 & 2 & 149 & ${ }_{1}^{2}$ & $\begin{array}{l}212 \\
213\end{array}$ & 1 \\
\hline $\begin{array}{l}39 \\
40\end{array}$ & $\begin{array}{l}1 \\
2\end{array}$ & $\begin{array}{l}93 \\
95\end{array}$ & ${ }_{1}^{2}$ & $\begin{array}{l}151 \\
152\end{array}$ & 1 & $\begin{array}{l}213 \\
215\end{array}$ & 6 \\
\hline & & & & 152 & 1 & 215 & 1 \\
\hline $40^{*}$ & 1 & 97 & 2 & $152^{*}$ & 9 & 217 & 2 \\
\hline 41 & 2 & 101 & 2 & 155 & 2 & 219 & 2 \\
\hline 44 & 2 & 103 & 1 & 156 & 2 & 220 & 2 \\
\hline 47 & 1 & 104 & 2 & 157 & 2 & 221 & 2 \\
\hline 51 & 2 & $104^{*}$ & 1 & 159 & 1 & 223 & 2 \\
\hline 52 & 2 & 105 & 2 & 161 & 2 & 227 & 2 \\
\hline 53 & 3 & 107 & 2 & 164 & 1 & & \\
\hline
\end{tabular}

TABLE 3. Talues of $k$ and corresponding combinations and values of $r$

\begin{tabular}{|c|c|c|}
\hline$k$ & Combination & $r$ \\
\hline $\begin{array}{r}43 \\
67 \\
88 \\
123 \\
148 \\
173 \\
187 \\
188 \\
197\end{array}$ & $\begin{array}{l}\left(1+2^{-s}\right)\left(1+3^{-s}\right) L(s, \chi) \\
\left(1+2^{-s}\right)\left(1+3^{-s}\right)\left(1+5^{-s}\right) L(s, \chi) \\
\left(1+3^{-s}\right) L(s, \chi) \\
\left(1+2^{-s}\right)\left(1+5^{-s}\right) L(s, \chi) \\
\left(1+3^{-s}\right)\left(1+5^{-s}\right)\left(1+7^{-s}\right) L(s, \chi) \\
\left(1+2^{-s}\left(1+3^{-s}\left(1+5^{-s}\right) L(s, \chi)\right.\right. \\
\left(1+2^{-s}\right)\left(1+3^{-s}\right)\left(1+5^{-s}\right) L(s, \chi) \\
\left(1+3^{-s}\right) L(s, \chi) \\
\left(1+2^{-s}\right) L(s, \chi)\end{array}$ & $\begin{array}{l}2 \\
3 \\
4 \\
2 \\
3 \\
4 \\
3 \\
3 \\
3 \\
2\end{array}$ \\
\hline
\end{tabular}

\section{References}

[1] J. Barkley Rosser, Real roots of Dirichlet L-series, Bul. Am. Math. Soc. 55, 906 to 913 (1949).

[2] E. Landau, Handbuch der Lehre von der Verteilung der Primzahlen, 1, Leipzig, Teubner, 1909.

[3] S. Chowla, Note on Dirichlet's L-functions, Acta Arithmetica, 1, 113 to 114 (1935).

[4] H. Heilbronn, On real characters, Acta Arithmetica, 2, 212 to 213 (1937).

[5] E. T. Whittaker and G. N. Watson, A course of modern analysis, American Edition (The Macmillan Co., New York, N. Y., 1947).

[6] E. Landau, Vorlesungen über zahlentheorie, 1, Leipzig, Hirzel, 1927.

[7] S. Chowla and A. Selberg, On Epstein's Zeta Function (I), Proc. Nat. Acad. of Sciences, 35, 371 to 374 (1949)

[8] H. Heilbronn, On Dirichlet series which satisfy a certain functional equation, Quart. Jour. of Math., Oxford series, $\mathbf{9}, 194$ to 195 (1938).

Los Angeles, January 11, 1950.

\title{
Forced Oscillations in Nonlinear Systems
}

\section{By Mary L. Cartwright}

\begin{abstract}
This paper shows how the approximate form of the solutions of a certain nonlinear differential equation occurring in radio work may be obtained from certain general results and gives the proof of the general results in detail. The proof of the general statement depends on a type of method that can be applied with minor modifications to any equation of the type

$$
\ddot{x}+k f(x) \dot{x}+g(x)=k p(t),
$$
\end{abstract}

where $p(t)$ has period $2 \pi / \lambda$, and $\int_{0}^{t} p(t) d t$ is bounded for all $t, f(x) \geq 1$ for $|x| \geq a$, and $g(x) / x \geq 1$ for $|x| \geq a$.

For some years Professor J. E. Littlewood and I have been working on nonlinear differential equations ${ }^{2}$ of a type which occur in radio work and elsewhere. One of the most interesting of these equations is

$$
\ddot{x}=k\left(1-x^{2}\right) \dot{x}+x-b k \lambda \cos (\lambda t+\alpha),
$$

especially for $k$ large and $0<b<2 / 3$. Our attention was drawn to it by a remark of van der $\mathrm{Pol},{ }^{3}$ which

1 This paper contains material presented in lecture form to the staff of the Institute for Numerical Analysis of the National Bureau of Standards on January 28,1949 . Miss Cartwright was a consultant at the INA at the time this lecture

was delivered.

2 See M. L. Cartwright and J. E. Littlewood, J. London Math. Soc. 20, 180
189 (1945), and Ann. Math. 48, 472-494 (1947); also M. L. Cartwright, J. Inst.

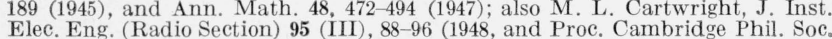
45, 495 (1949)

3 B. van der Pol, Proc. Inst. Radio Eng. 22, 1051-1086 (1934). suggested that it corresponded to a physical system investigated by him and van der Mark. ${ }^{4}$ For certain values of the parameters the physical system had two possible stable oscillations, one of period $4 n \pi / \lambda$ and one of period $(2 n+1) 2 \pi / \lambda$. As a matter of fact in the case of (1), owing to the strictly symmetrical nonlinear function $1-x^{2}$, the period $4 n \pi / \lambda$ does not occur, but for certain values of $b$ there are two stable oscillations of periods $(2 n \pm 1) 2 \pi / \lambda$.

It would take too long to give a complete proof of this statement here, but I propose to show how the approximate form of the solutions may be obtained from certain very general results, and give the proof of the general results in detail. The proof

4 B. van der Pol and J. van der Mark, Nature 120, 333-364 (1927) 